\title{
Evaluation of screening methods for identification of patients with chronic rheumatological disease requiring tuberculosis chemoprophylaxis prior to commencement of TNF- $\alpha$ antagonist therapy
}

\author{
Aran Singanayagam, ${ }^{1}$ Kavina Manalan, ${ }^{1}$ Saranya Sridhar, ${ }^{1}$ Philip L Molyneaux, ${ }^{1}$ \\ David W Connell, ${ }^{1}$ Peter M George, ${ }^{1}$ Anne Kindelerer, ${ }^{2}$ Suranjith Seneviratne, ${ }^{3}$ \\ Ajit Lalvani, ${ }^{1}$ Melissa Wickremasinghe, ${ }^{1}$ Onn Min Kon ${ }^{1}$
}

- Additional material is published online only. To view please visit the journal online (http://dx.doi.org/10.1136/ thoraxjnl-2013-203436).

${ }^{1}$ Chest and Allergy Department, St Mary's Hospital, Imperial College NHS trust, London, UK

${ }^{2}$ Department of Rheumatology, St. Mary's Hospital, Imperial College NHS Trust, London, UK ${ }^{3}$ Department of Clinical Immunology, St. Mary's Hospital, Imperial College NHS Trust, London, UK

\section{Correspondence to} Dr Melissa Wickremasinghe, Chest and Allergy Department, St Mary's Hospital, Imperial College NHS trust, London W2 1NY, UK; melissa. wickremasinghe@imperial. nhs.uk

MW and OMK joint last authors.

Published Online First 23 August 2013

To cite: Singanayagam $A$, Manalan K, Sridhar S, et al. Thorax 2013;68:955-961.

\section{ABSTRACT}

Background Patients undergoing tumour necrosis factor (TNF)- $\alpha$ antagonist therapy are at increased risk of latent tuberculosis infection (LTBI) reactivation. The aim of this study was to determine the optimum available screening strategy for identifying patients for tuberculosis (TB) chemoprophylaxis.

Methods We conducted a prospective observational study of consecutive adults with chronic rheumatological disease referred for LTBI screening prior to

commencement of TNF- $\alpha$ antagonist therapy. All patients included had calculation of TB risk according to age, ethnicity and year of UK entry, as described in the 2005 British Thoracic Society (BTS) guidelines and measurement of tuberculin skin test (TST) and T.Spot.TB. Results There were 187 patients included in the study, with 157 patients (84\%) taking immunosuppressants. 137 patients would require further risk stratification according to the BTS algorithm, with $110(80.3 \%)$ classified as being at low risk of having LTBI. There were 39 patients (35.5\%) who were categorised as low risk but were either TST and/or T.Spot positive and would not have received chemoprophylaxis according to the BTS algorithm. Combination of all three methods (risk stratification and/or positive T.Spot and/or positive TST) identified 66 patients out of 137 who would potentially be offered chemoprophylaxis, which was greater than any single test or two-test combination.

Conclusion Performing both a TST and T.Spot in patients on immunosuppressants prior to commencement of TNF- $\alpha$ antagonist therapy gives an additional yield of potential LTBI compared with use of risk stratification tables alone. Our results suggest that use of all three screening modalities gives the highest yield of patients potentially requiring chemoprophylaxis.

\section{INTRODUCTION}

Tumour necrosis factor (TNF)- $\alpha$ inhibitors are frequently used to treat immune-mediated inflammatory diseases and are recommended for a range of rheumatological conditions. However, patients undergoing TNF- $\alpha$ inhibitor therapy are at increased risk of developing serious opportunistic infections, including reactivation of latent tuberculosis infection (LTBI). ${ }^{1-3}$ Crucially, treatment with chemoprophylaxis in patients who are diagnosed with LTBI prior to commencement of TNF- $\alpha$

\section{Key messages}

What is the key question?

- What is the optimum screening method for identification of patients who require tuberculosis (TB) chemoprophylaxis prior to commencement of tumour necrosis factor- $\alpha$ (TNF- $\alpha$ ) antagonist therapy?

What is the bottom line?

- Performing both a tuberculin skin test (TST) and T.Spot in patients on immunosuppressants prior to commencement of TNF- $\alpha$ antagonist gives an additional yield of potential latent tuberculosis infection compared with use of risk stratification tables or either test alone.

Why read on?

- This study aims to determine the optimum screening strategy, and this is of clinical importance, given that there is significant increased risk of TB reactivation associated with use of TNF- $\alpha$ antagonist therapy.

inhibitor therapy is associated with a $74 \%$ reduction in risk of tuberculosis (TB) reactivation. ${ }^{4}$

Screening for LTBI prior to initiation of TNF- $\alpha$ inhibitors is now part of routine care in the UK, and specific guidance exists in the form of the 2005 British Thoracic Society (BTS) guidelines. ${ }^{5}$ The optimum screening strategy remains unclear with different countries recommending varying approaches based on using risk stratification as a surrogate for LTBI and/or one or a combination of interferon- $\gamma$ release assay (IGRA) and tuberculin skin testing (TST). ${ }^{2}{ }^{6-10}$ The BTS recommend a specific algorithm and advocate sole use of risk stratification tables based on age, ethnicity and year of UK entry to guide chemoprophylaxis decisions for patients on immunosuppressant therapy due to the fact that the TST may be falsely negative in the setting of immunosuppressants. ${ }^{11}$ The guidelines recommend chemoprophylaxis decisions based on balancing the population risk of LTBI in different groups using age and ethnicity with the risk of therapy-induced serious hepatotoxicity, and 
assume equivalence between the significance of the two potential complications of LTBI reactivation and hepatotoxicity. Additionally, since the publication of the BTS guidance, routine IGRA testing is now becoming more widely available to clinicians as an alternative method of assessing for LTBI.

A number of previous studies have assessed the correlation between IGRAs and TST in LTBI screening prior to TNF- $\alpha$ antagonist therapy, ${ }^{12-16}$ but no study has directly compared the performance of these tests with the risk stratification method advocated by the BTS. Only a small number of studies have taken into account whether the patient is on immunosuppressant medication. ${ }^{17} 18$ At our centre, we adopt an alternative unique approach of undertaking 'triple testing' (combined BTS risk stratification, TST and T.Spot TB) in all patients referred for LTBI screening prior to commencement of TNF- $\alpha$ antagonist therapy. The aim of our study was, therefore, to compare the performance of the BTS risk stratification approach with use of the T. Spot and/or TST in order to determine the optimal screening strategy in patients with rheumatological disease being considered for TNF- $\alpha$ inhibitor therapy.

\section{METHODS}

We conducted a prospective observational study of consecutive adult patients( $>16$ years) with chronic rheumatological disease referred for LTBI screening prior to commencement of TNF- $\alpha$ inhibitor therapy at St Mary's Hospital, London, between September 2009 and September 2012. The study was approved by the Local Joint Research Office.

Information about demographics, including age, ethnicity, rheumatological diagnosis, Bacille Calmette-Guerin (BCG) vaccination status and, if appropriate, year of entry to the UK, were recorded on a standardised pro forma. A clinical history was taken from all patients with specific enquiry about symptoms of active TB (cough, fever, weight loss and night sweats) and drug history including current use of immunosuppressant medications. Enquiry was also made about previous history of active $\mathrm{TB}$ and treatment received and any previous close contact with TB-infected persons was recorded, as previously described. ${ }^{10}$

All patients had calculation of TB risk according to age, ethnicity and year of UK entry, as described in the 2005 BTS guidelines (see online supplementary material for details). A chest radiograph to assess for signs of active TB or previous TB (eg, apical scarring, calcified granulomata) was also carried out.

\section{Interferon $\gamma$ ELISPOT and TST}

The T.Spot.TB test (Oxford Immunotech, Oxford UK) was measured as previously described. ${ }^{19}$ Peripheral venous blood samples were collected and assays were carried out in accordance with the manufacturer's instructions. Results were defined as positive, negative or indeterminate depending on the manufacturer's criteria.

The TST was undertaken, according to the Mantoux method, by trained TB specialist nurses. A $0.1 \mathrm{~mL}$ dose (2 IU) of tuberculin purified protein derivative (PPD RT23; Staten Serum Institute, Copenhagen, Denmark) was injected intradermally, according to standard guidelines. The main diameter of skin induration was then recorded $48-72 \mathrm{~h}$ after inoculation and result was recorded. A transverse diameter of $\geq 6 \mathrm{~mm}$ in the absence of previous BCG vaccination or $\geq 15 \mathrm{~mm}$ in the presence of previous BCG vaccination was considered positive. Patients who had no visible BCG scar and were unable to recall history of receiving BCG were assumed to have not received it.

\section{Exclusion criteria}

Patients who did not receive TST and T.Spot tests, or in whom either test was declined or not successfully completed were excluded from the study.

\section{Statistical analysis}

All data were analysed using SPSS 13.0 for windows (Chicago, Illinois, USA). The $\chi^{2}$ test was used to compare the proportions of factors of interest in patients categorised as high risk versus low risk, patients with positive T.Spot versus those with negative T.Spot, and patients with positive TST versus those with negative TST. The Mann-Whitney U test was used to compare the median age as a continuous variable between patients categorised as high risk versus low risk, patients with positive T.Spot versus those with negative T.Spot and patients with positive TST versus those with negative TST. All analyses were undertaken using two-tailed tests with a $\mathrm{p}<0.05$ considered to be statistically significant.

\section{RESULTS}

There were 187 patients included in the study. Figure 1 shows patient entry into the study. Baseline demographics of the study population are shown in table 1. There were 157 patients $(84.0 \%)$ taking immunosuppressants at the time of LTBI screening. There was a non-statistically significant trend towards patients on immunosuppressants being less likely to have positive TST results $(40.0 \%$ vs $22.9 \% ; \mathrm{p}=0.09)$, but no differences between the proportion of patients who were high risk according to BTS risk tables or who were T.Spot positive (etable 1, see online supplement).

\section{EVALUATION OF RISK STRATIFICATION ALONE AS A METHOD OF IDENTIFYING PATIENTS REQUIRING CHEMOPROPHYLAXIS}

The BTS advocates use of a risk-stratification approach alone for patients who are on immunosuppressants, and therefore, we assessed the yield of this method. Of the subgroup on immunosuppressants, 20 patients had either previous history ofTB and/ or chest radiograph consistent with prior $\mathrm{TB}$, and/or were diagnosed with active TB at the time of screening. This left a total of 137 patients who, according to the BTS algorithm, would require further risk stratification. Two patients had a history of previous contact with an active TB case. Of the subgroup on immunosuppressants requiring further risk stratification, 27 $(19.7 \%)$ patients were categorised as high risk. Table 2 shows a comparison between patients classified as high and low risk.

There were 26 out of 110 patients (23.6\%) who were categorised as low risk, but had a positive TST. Twenty-six out of 31 $(83.9 \%)$ of all patients with positive TST were categorised as low risk and would not have received chemoprophylaxis according to the BTS algorithm. In these low-risk patients with a positive TST, the median TST reaction size was $20 \mathrm{~mm}$ (15.5-20.5 mm). Fourteen out of $26(53.8 \%)$ patients had strongly positive TST ( $\geq 20 \mathrm{~mm}$ ). Of these 14 patients, nine $(64.3 \%)$ had been previously vaccinated with BCG. There were 21 out of 110 patients (19.1\%) who were categorised as low risk according to the BTS but had a positive T.Spot; 21 out of $25(84.0 \%)$ of all patients with positive T.Spot were categorised as low risk and would not have received chemoprophylaxis according to the BTS algorithm.

In total, there were 39 patients $(35.5 \%)$ who were categorised as low risk but had either TST and/or T.Spot positive and would not have received chemoprophylaxis according to the BTS algorithm. 
Figure 1 Flow chart of patient entry into study. Abbreviations: BTS, British Thoracic Society; CXR, chest radiograph; TB, tuberculosis; TNF, tumour necrosis factor; TST, tuberculin skin test.

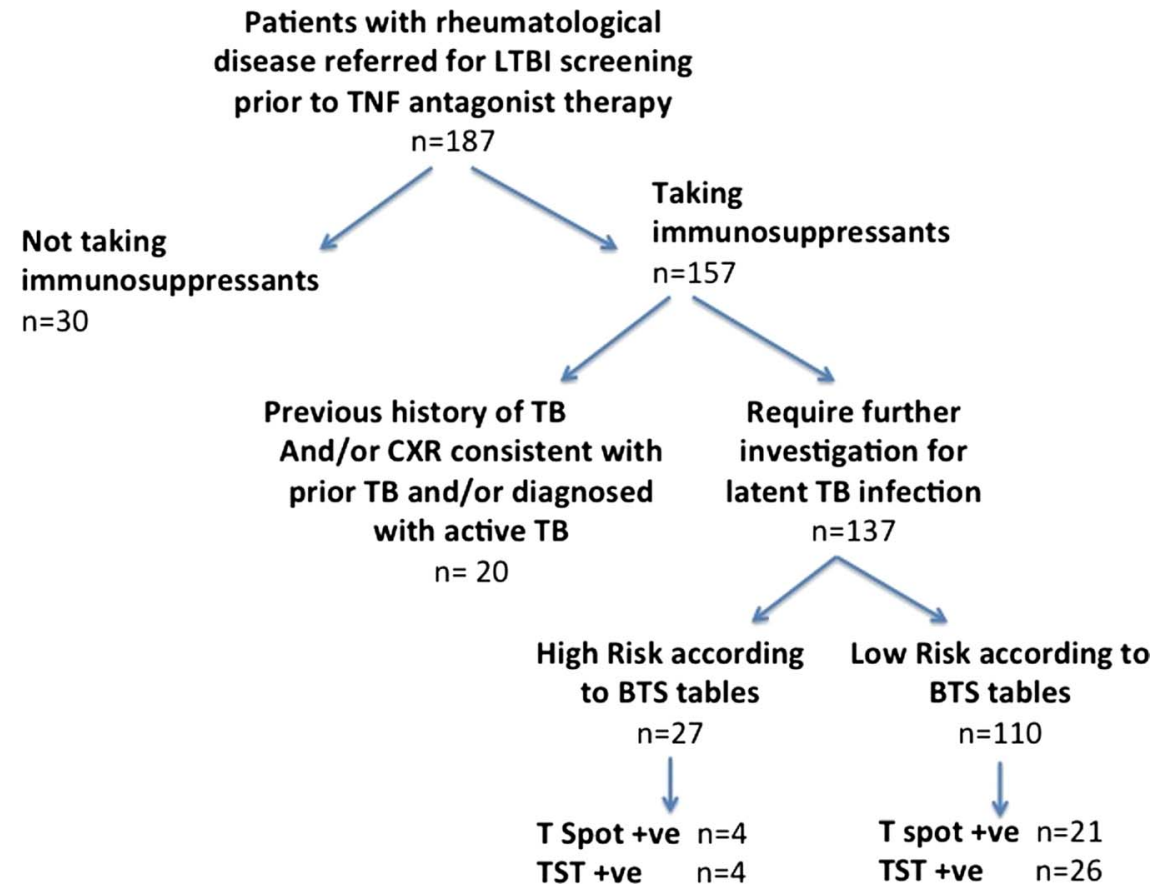

All three 'double test' combinations identified more patients for chemoprophylaxis than the remaining test alone. The best 'double test' combination method was risk stratification plus TST with a higher sensitivity than risk stratification and T.Spot combined or T.Spot and TST combined. Combination of all three methods identified 66 patients (48.2\%) who would potentially be offered chemoprophylaxis, which was greater than any single test or any two-test combination. Figure 2 shows overlap between the yields for each test individually and the number of patients who were double and triple test positive (ie, both or all three tests mutually positive).

\section{USE OF T.SPOT AND/OR TST IN LOW-RISK PATIENTS}

Given that high-risk patients on immunosuppressants are recommended to receive treatment by the BTS algorithm without requirement for measurement of TST or T.Spot, we evaluated the yield of these tests in the subgroup classified as low risk $(n=110)$. Again, TST identified more patients and missed fewer than T.Spot with double combination giving the highest yield (see table 4).

\section{CLINICAL OUTCOMES IN PATIENTS WHO RECEIVED CHEMOPROPHYLAXIS}

All patients who had positive results according to one or more of the three methods used $(n=66)$ were offered chemoprophylaxis in the form of a 3-month course of rifampicin plus isoniazid (or a 6-month course of isoniazid alone if on maintenance steroids), as per BTS guidelines. ${ }^{5}$ One patient declined treatment, leaving a total of 65 patients who received treatment. Of these, two patients $(3.1 \%)$ developed drug-induced hepatotoxicity requiring conversion to an alternative agent and three patients $(4.6 \%)$ required alternative agents for other intolerance to therapy. There were no other therapy-related complications observed in the study.

\section{DISCUSSION}

Our study is the first to compare a risk-stratification method of identifying patients requiring chemoprophylaxis with tests to 
Table 1 Baseline characteristics of study population

Total cohort ( $\mathrm{n}=187$ patients)

$\begin{array}{lc}\text { Demographics } & \\ \text { Age (median (IQR)) } & 54(43-65) \\ \text { Male sex } & 82(43.9 \%) \\ \text { Ethnicities } & \\ \text { Caucasian } & 109(58.3 \%) \\ \text { Black African } & 13(7.0 \%) \\ \text { Asian } & 23(12.3 \%) \\ \text { Other } & 42(22.5 \%) \\ \text { Comorbidities } & \\ \text { Chronic lung disease } & 13(7.0 \%) \\ \text { Ischaemic heart disease } & 8(4.3 \%) \\ \text { Diabetes mellitus } & 10(5.3 \%) \\ \text { HIV } & 1(0.5 \%) \\ \text { Inflammatory bowel disease } & 3(1.6 \%) \\ \text { Rheumatological diagnoses* } & \\ \text { Rheumatoid arthritis } & 113(60.4 \%) \\ \text { Ankylosing spondylitis } & 38(20.3 \%) \\ \text { Psoriatic arthropathy } & 23(12.3 \%) \\ \text { Juvenile idiopathic arthritis } & 2(1.1 \%) \\ \text { Other } & 13(7.0 \%) \\ \text { Immunosuppressants } \dagger & \\ \text { Methotrexate } & 113(60.4 \%) \\ \text { Azathioprine } & 3(1.6 \%) \\ \text { Sulphasalazine } & 46(24.6 \%) \\ \text { Leflunomide } & 13(7.0 \%) \\ \text { Maintenance steroids } & 47(25.1 \%) \\ \text { Other } & 1(0.5 \%)\end{array}$

LTBI screening

BCG vaccinated $\quad 65(34.8 \%)$

T.Spot positive $\quad 42(22.5 \%)$

T.Spot indeterminate $\quad 1(0.5 \%)$

TST positive $\quad 48(25.7 \%)$

T.Spot and TST both positive $\quad 18(9.6 \%)$

High risk according to BTS risk tables $\quad 40(21.4 \%)$

CXR suggestive of previous TB $16(8.6 \%)$

Contact of active TB case $\quad 8(4.3 \%)$

Previously treated for TB $2(1.1 \%)$

Diagnosed with active TB $2(1.1 \%)$

Data presented as $n$ (\% of total cohort).

*Total exceeds 187 due to some patients having dual diagnoses.

†Total exceeds 187 due to some patients being on more than one

immunosuppressant agent.

BCG, Bacille Calmette-Guer; BTS, British Thoracic Society; CXR, chest radiograph;

LTBI, latent tuberculosis infection; TB, tuberculosis; TST, tuberculin skin test.

directly identify LTBI prior to TNF- $\alpha$ inhibitor therapy. Our data show that use of risk-factor tables alone to guide treatment in patients taking immunosuppressants, as recommended by the BTS guidelines, misses a large number of predominantly Caucasian patients who have apparent LTBI as defined by a positive TST or T.Spot. Although deemed to be 'low risk' based on age and ethnicity, a total of 39 patients $(35.5 \%)$ in this category had evidence of LTBI according to TST and/or T.Spot and these patients would not have been treated according to the BTS guidance.

We evaluated the yield of each test individually and also the yield of a combination of either (or all three) tests being positive. Use of TST alone identified slightly more patients with apparent LTBI with a higher sensitivity than either of the other tests alone, which is in keeping with some previous studies. ${ }^{13} 14$
Use of combinations of any two methods gave higher yields than the missed yield from the remaining test. Overall, use of all three tests (any one or more being positive) led to the greatest yield of patients identified for treatment. Triple testing was performed in all patients in the current study. However, measurement of TST and T.Spot in those classified as high risk and on immunosuppression is unlikely to alter management, except to aid drug compliance and, in reality, such patients would be offered chemoprophylaxis regardless of the results of these tests. Therefore, in clinical practice, the most cost-effective strategy may be two-step: risk stratification in all with chemoprophylaxis offered to those who are high risk, followed by measurement of both T.Spot and TST in all low-risk patients (see online supplementary material for proposed alternative screening algorithm based on the results of this study). Prospective independent validation of this strategy is now required. It is also notable that in clinical practice, many centres are now using IGRA testing alone in preference to TST. According to our data, use of risk stratification followed by IGRA testing in low-risk patients would have a lower sensitivity for identification of patients requiring chemoprophylaxis than dual testing with IGRA and TST. However, given that the risk of reactivation in older patients with positive TST is likely to be low, dual testing with risk stratification followed by IGRA may still be an acceptable alternative strategy. Further prospective evaluation is required.

Our data shows low correlation between traditional risk factors for LTBI (age, ethnicity and UK year of entry) and T. Spot and TST positivity. We found that $86.7 \%$ of all patients on immunosuppressants with positive TST, and $84 \%$ with positive T.Spot were classified as low-risk according to the BTS risktables. This result was surprising as we would have expected a higher correlation, given that risk-stratification alone is the method recommended by the BTS for guiding chemoprophylaxis in these patients. The precise reasons for poor correlation in the high-risk group are unclear, although we speculate that it may be due to the effects of rheumatological disease processes and/or immunosuppressant medication perhaps reducing the likelihood of T.Spot or TST positivity in these high-risk ethnic groups.

Our data suggests that Caucasian patients may be wrongly denied chemoprophylaxis, given their automatic classification as low risk by the BTS risk tables. Of our cohort, 58.3\% were of Caucasian ethnicity, but despite this, many had a positive T.Spot and/or TST. It is our belief that a Caucasian individual who has underlying immunosuppression due to medication and/or rheumatological disease cannot be considered to have an equivalent risk of acquisition of LTBI to a normal, immunocompetent Caucasian individual. Consequently, use of risk-factor tables based on ethnicity may not be an appropriate strategy to guide chemoprophylaxis in this patient cohort.

Matulis and colleagues previously assessed correlation between TST and Quantiferon with risk factors for LTBI in patients with rheumatologic disease on immunosuppressants. ${ }^{17}$ Similarly, they found a number of patients who were low risk but had positive IGRA (12\%) or TST (57\%). One possible explanation for these results is that in a low-prevalence population, the false positive rate of a test may increase and we do not yet have prospective longitudinal data to understand the clinical significance of positive T.Spot results in this specific cohort. However, it is notable that the regional endemic rate of TB in London is considerably higher than national UK rates, ${ }^{21}$ and this may contribute to the higher than expected frequency of LTBI seen in low-risk patients in our cohort. Risk-stratification 
Table 2 Comparison of characteristics in patients on immunosuppressants classified according to risk stratification, tuberculin skin test (TST) positivity and T.Spot positivity

\begin{tabular}{|c|c|c|c|c|c|c|c|c|c|}
\hline & $\begin{array}{l}\text { High risk } \\
\text { according to BTS } \\
n=27\end{array}$ & $\begin{array}{l}\text { Low risk } \\
\text { according to BTS } \\
n=110\end{array}$ & $\begin{array}{l}p \\
\text { Value }\end{array}$ & $\begin{array}{l}\text { TST positive } \\
\mathrm{n}=31\end{array}$ & $\begin{array}{l}\text { TST negative } \\
n=106\end{array}$ & p Value & $\begin{array}{l}\text { T.Spot } \\
\text { positive } \\
n=25\end{array}$ & $\begin{array}{l}\text { T.Spot negative } \\
\mathrm{n}=111^{*}\end{array}$ & p Value \\
\hline Male sex (\%) & $15(55.6)$ & $45(40.9)$ & 0.197 & $18(58.1)$ & $42(39.6)$ & 0.099 & $14(56.0)$ & $46(41.4)$ & 0.265 \\
\hline Age & $50(44-60)$ & $57(45-68)$ & 0.063 & 57 (49-68) & $55(44-65)$ & 0.530 & $56(46-68)$ & $55.5(44-66)$ & 0.624 \\
\hline \multicolumn{10}{|l|}{ Ethnicity } \\
\hline Caucasian (\%) & $0(0)$ & $83(75.5)$ & $<0.001$ & $19(61.3)$ & $64(60.4)$ & 1.0 & $16(64.0)$ & $67(60.4)$ & 0.823 \\
\hline Black African (\%) & $8(29.6)$ & $0(0)$ & $<0.001$ & $2(6.5)$ & $6(5.7)$ & 1.0 & $3(12.0)$ & $5(4.5)$ & 0.163 \\
\hline Asian (\%) & $15(55.6)$ & $0(0)$ & $<0.001$ & $3(9.7)$ & $12(11.3)$ & 1.0 & $1(4.0)$ & $14(12.6)$ & 0.304 \\
\hline Other $(\%)$ & 4 (14.8) & $27(24.5)$ & 0.441 & $7(22.6)$ & $24(22.6)$ & 1.0 & $5(20.0)$ & $25(22.5)$ & 1.0 \\
\hline $\begin{array}{l}\text { High risk according to } \\
\text { BTS }(\%)\end{array}$ & - & - & - & $5(16.1)$ & $22(20.8)$ & 0.798 & $4(16.0)$ & $23(20.7 \%)$ & 0.783 \\
\hline TST positive (\%) & $4(14.8)$ & $26(23.6)$ & 0.439 & - & - & - & $9(36.0)$ & $22(19.8)$ & 0.112 \\
\hline T.Spot positive (\%) & $4(14.8)$ & $21(19.1)$ & 0.783 & $9(29.0)$ & $16(15.1)$ & 0.110 & - & - & - \\
\hline $\begin{array}{l}\text { Indeterminate T.Spot } \\
(\%)\end{array}$ & $0(0)$ & $1(0.9)$ & 1.0 & - & - & - & - & - & - \\
\hline $\begin{array}{l}\text { T.Spot and TST both } \\
\text { positive (\%) }\end{array}$ & $1(3.7)$ & $8(7.3)$ & 0.688 & - & - & - & - & - & - \\
\hline $\begin{array}{l}\text { Either T.Spot and/or } \\
\text { TST positive (\%) }\end{array}$ & $7(25.9)$ & $39(35.5)$ & 0.496 & - & - & - & - & - & - \\
\hline
\end{tabular}

*Total=136 due to one patient having indeterminate T.Spot result.

Data presented as median (IQR) or $\mathrm{n}(\%)$; $\mathrm{p}$ values show comparison of proportions between groups or median values for continuous variables.

BTS, British Thoracic Society.

tables based on national statistics such as those advocated in the BTS guideline may, therefore, not be applicable to regions with higher endemic TB rates. Interestingly, the BTS document does not discuss prior contact with an active TB case as a possible risk factor for LTBI. In our study, only two patients in the subgroup on immunosuppressants gave a history of prior contact with an active TB case and both these patients were classified as high risk on basis of risk-factor tables. It may be possible that a greater number of patients in the low-risk category had been exposed to an active TB case without their knowledge, and this may explain the relatively high TST and T.Spot positivity in so-called 'low risk' individuals.

We found a trend towards TST being less likely to be positive in patients on immunosuppressants compared with those who were not. Use of the TST as a modality for identifying LTBI in immunosuppressed patients is not advocated by the BTS guidelines, since previous studies have suggested the possibility of false negative results. ${ }^{1122}$ Previous studies have suggested that IGRAs, such as T.Spot, may be less affected by immune suppression, specifically in the context of HIV-infected patients, ${ }^{23}$ and may also correlate better with LTBI risk factors in patients with inflammatory disorders taking immnosuppressants. ${ }^{17}$ In keeping with this, we found that T.Spot positivity was unaffected by immunosuppression in our cohort.

We still observed 36 patients (22.9\%) who had a positive TST result despite immunosuppressant therapy. A potential known confounder of the TST is the possibility of false positive results, particularly in those with prior BCG vaccination or exposure to environmental mycobacteria. ${ }^{24}{ }^{25}$ However, patients classified as low risk, according to the BTS tables, should be expected to

Table 3 Evaluation of yield of each test individually and combinations of tests

\begin{tabular}{|c|c|c|c|}
\hline Approach & $\begin{array}{l}\text { Number of patients identified for } \\
\text { chemoprophylaxis (\%) }\end{array}$ & $\begin{array}{l}\text { Number of patients missed (would have been } \\
\text { identified via other methods) (\%) }\end{array}$ & $\begin{array}{l}\text { Sensitivity versus triple } \\
\text { combination testing (\%) }\end{array}$ \\
\hline \multicolumn{4}{|l|}{ Single test } \\
\hline Risk factor approach alone & $27(19.7)$ & $39(28.5)$ & $40.9(29.0-53.7)$ \\
\hline T.Spot positive alone & $25(18.2)$ & $41(29.9)$ & $37.9(26.2-50.7)$ \\
\hline TST positive alone & $31(22.6)$ & $35(25.5)$ & $47.0(34.6-59.7)$ \\
\hline \multicolumn{4}{|l|}{ Double test combinations } \\
\hline $\begin{array}{l}\text { Risk factor and/or T.Spot } \\
\text { positive }\end{array}$ & $48(35.0)$ & $18(13.1)$ & $72.7(60.4-83.0)$ \\
\hline Risk factor and/or TST positive & $53(38.7)$ & $13(9.5)$ & $80.3(68.7-89.1)$ \\
\hline $\begin{array}{l}\text { T.Spot positive and/or TST } \\
\text { positive }\end{array}$ & $47(34.3)$ & 19 (13.9) & $71.2(58.7-81.7)$ \\
\hline \multicolumn{4}{|l|}{ Triple test combination } \\
\hline $\begin{array}{l}\text { Risk factor and/or T.Spot } \\
\text { positive and/or TST positive }\end{array}$ & $66(48.2)$ & - & - \\
\hline
\end{tabular}




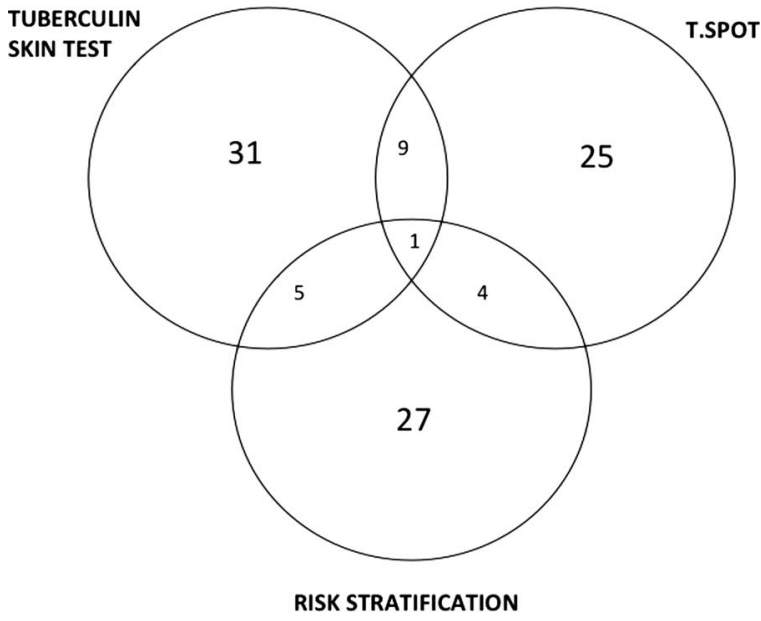

Figure 2 Overlapping yield for tests when mutually positive.

have low rates of TST positivity and, as previously discussed, the increased rate of positivity may be explained by higher regional endemic rates of $\mathrm{TB}$ in London than the national $\mathrm{UK}$ rates.

Previous studies have correlated increasing sizes of Mantoux reaction to increased risk of TB reactivation. ${ }^{26}$ Furthermore, any TST positivity in the context of immunosuppression may be considered relevant. ${ }^{9}$ Studies have also shown that IGRAs have a higher positive and negative predictive value for progression to active TB compared with the TST. ${ }^{27} 28$ To date, the risk of reactivation according to TST or IGRA results, specifically in immunosuppressed patients, has not been extensively evaluated prospectively, except for one small study in patients with HIV. ${ }^{29}$ There are no existing studies evaluating the predictive value of the BTS risk factor approach for LTBI reactivation.

In our low-risk subgroup, the median TST reaction size was $20 \mathrm{~mm}$, with 14 out of 26 patients having strongly positive $(\geq 20 \mathrm{~mm})$ results. Of these 14 patients, 5 (35.7\%) had no history of previous BCG vaccination. It is difficult to justify giving these patients TNF- $\alpha$ antagonist therapy without prior treatment with chemoprophylaxis, despite the poor correlation with T.Spot positivity in this group. Some guidelines advocate use of lower TST-positive thresholds (eg, $>5 \mathrm{~mm}$ regardless of prior BCG in the context of immunosuppression. ${ }^{9}{ }^{20}$ ) This would have led to an even greater number of patients requiring therapy in our cohort (additional 25 patients). Our approach is to use standard thresholds with correction for prior BCG, to try

Table 4 Evaluation of yield of T.Spot and tuberculin skin test (TST) in patients categorised as low risk

\begin{tabular}{|c|c|c|c|}
\hline & $\begin{array}{l}\text { Number of patients } \\
\text { identified for } \\
\text { chemoprophylaxis } \\
(\%)\end{array}$ & $\begin{array}{l}\text { Number of } \\
\text { patients } \\
\text { missed (would } \\
\text { have been } \\
\text { identified via } \\
\text { other } \\
\text { methods) (\%) }\end{array}$ & $\begin{array}{l}\text { Sensitivity } \\
\text { versus double } \\
\text { testing } \\
\text { combination } \\
(\%)\end{array}$ \\
\hline T.Spot +ve & $21(19.1)$ & $18(16.4)$ & $53.8(37.2-69.9)$ \\
\hline TST +ve & $26(23.6)$ & $13(11.8)$ & $66.7(49.8-80.9)$ \\
\hline $\begin{array}{l}\text { T.Spot and/or } \\
\text { TST + ve }\end{array}$ & $39(35.5)$ & - & - \\
\hline
\end{tabular}

Data shown as $\mathrm{n}(\%$ of low risk sub-group 110$)$ or sensitivity $(95 \% \mathrm{Cl})$. and reduce the number of patients treated unnecessarily for false positive results. However, if either risk stratification or T.Spot is positive in the context of a negative TST according to these thresholds, we recommend chemoprophylaxis.

Overall, we advocate a two-step triple testing approach to screening prior to TNF $\alpha$ antagonist therapy, given that it identifies more patients requiring chemoprophylaxis than any other single test or double combination method. Similar strategies have previously been postulated, ${ }^{30-32}$ and our study offers supportive data for such approaches. Triple testing offers increased sensitivity to detect potential cases of LTBI and, although false positives may be more likely, we have no prognostic data for these tests in this setting. We believe that the risk associated with LTBI reactivation far outweighs the potential risks associated with therapy-induced hepatotoxicity, especially if adequate monitoring is in place. ${ }^{33}$ The BTS guidelines assume an equivalence of clinical significance between these two complications. Notably, despite a higher number of patients being treated with chemoprophylaxis in our study than by recommended algorithms, we only observed treatment-limiting hepatotoxicity in two patients $(3.1 \%)$. This is comparable with rates of drug-induced hepatotoxicity reported by other studies of rifampicin and isoniazid chemoprophylaxis for LTBI, ${ }^{34}$ and therefore, in the current study, our strategy did not appear to lead to significantly increased risks of hepatotoxicity. Previous studies have also reported low rates of drug-induced hepatotoxicity in a similar setting. ${ }^{35}$

Our study has limitations. First, the absence of a 'gold standard' for the diagnosis of LTBI makes it impossible to determine sensitivities and specificities for the screening methods evaluated, and the prognostic values of the risk factor approach, TST and IGRA in the setting of immunosuppression are unclear. A prospective follow-up study of patients classified as high or low risk according to the BTS tables is required for further evaluation. Second, our study has been carried out in an urban, ethnically diverse region and may not be applicable to other regions. Some authors advocate tailored screening strategies depending on the prevalence of TB disease and whether BCG is frequently used in the area. ${ }^{32}$ There are no existing studies that compare strategies in low-prevalence and high-prevalence areas.

In summary, performing a TST and T.Spot in patients on immunosuppressants prior to commencement of TNF- $\alpha$ antagonist therapy gives an additional yield of potential LTBI compared with use of risk stratification tables alone. Our results suggest that use of all three screening modalities gives the highest yield of patients potentially requiring chemoprophylaxis. Larger independent validation of this method is required along with development of more accurate composite risk-prediction models incorporating predictive biomarkers and other clinical risk factors.

Contributors Conception and design: AS, SaS, AK, AL, MW, OMK. Data collection: AS, KM, PLM, DWC, PMG, SUS, AK. Analysis and interpretation: AS, SaS, MW, OMK. Drafting and editing manuscript: all authors. OMK is guarantor for the study.

Funding This study was undertaken at St Mary's Hospital. Imperial College Healthcare NHS trust which is supported by the NIHR Biomedical Research Centre funding scheme and the Centre for Respiratory Infection at Imperial College London, supported by The Wellcome Trust.

Competing interests Professor Lalvani is inventor for patents underpinning T-cell-based diagnosis. The ESAT-6/CFP-10 IFN- $\gamma$ ELISpot assay was commercialised by an Oxford University spin-out company (T-SPOT.TB®, Oxford Immunotec Ltd, Abingdon, UK) in which the University of Oxford and Professor Lalvani have minority shares of equity and royalty entitlements.

Ethics approval Imperial College JRO.

Provenance and peer review Not commissioned; externally peer reviewed. 


\section{REFERENCES}

1 Keane J, Gershon S, Wise RP, et al. Tuberculosis associated with infliximab, a tumor necrosis factor alpha-neutralizing agent. N Engl J Med 2001;345:1098-104.

2 Solovic I, Sester M, Gomez-Reino JJ, et al. The risk of tuberculosis related to tumour necrosis factor antagonist therapies: a TBNET consensus statement. Eur Respir J 2010;36:1185-206.

3 Bongartz T, Sutton AJ, Sweeting MJ, et al. Anti-TNF antibody therapy in rheumatoid arthritis and the risk of serious infections and malignancies: systematic review and meta-analysis of rare harmful effects in randomized controlled trials. JAMA 2006;295:2275-85.

4 Carmona L, Gomez-Reino JJ, Rodriguez-Valverde V, et al. Effectiveness of recommendations to prevent reactivation of latent tuberculosis infection in patients treated with tumor necrosis factor antagonists. ArthritisRheum 2005;52:1766-72.

5 Ormerod LP, Milburn HJ, Gillspie S, et al. BTS recommendations for assessing risk and for managing Mycobacterium tuberculosis infection and disease in patients due to start anti-TNF-alpha treatment. Thorax 2005;60:800-5.

6 Mariette $X$, Salmon D. French guidelines for diagnosis and treating latent and active tuberculosis in patients with RA treated with TNF blockers. Ann Rheum Dis 2003:62:791.

7 American Thoracic Society and Centers for disease control and prevention (CDC). Targeted tuberculin testing and treatment of latent tuberculosis infection. This official statement of the American Thoracic Society was adopted by the ATS Board of Directors, July 1999. This is a Joint Statement of the American Thoracic Society (ATS) and the Centers for Disease Control and Prevention (CDC). This statement was endorsed by the Council of the Infectious Diseases Society of America. (IDSA), September 1999, and the sections of this statement. Am J Respir Crit Care Med 2000;161(4 Pt 2):S221-47.

8 Ding T, Ledingham J, Luqmani R, et al. BSR and BHPR rheumatoid arthritis guidelines on safety of anti-TNF therapies. Rheumatology (Oxford) 2010;49:2217-19.

9 Tuberculosis associated with blocking agents against tumor necrosis factor-alphaCalifornia, 2002-2003. MMWR Morb Mortal Wkly Rep 2004;53:683-6.

10 National Institute for Clinical Excellence (NICE). Tuberculosis: clinical diagnosis and management of tuberculosis, and measures for its prevention and control, 2011. http://guidance.nice.org.uk/CG117/NICEGuidance/pdf/English (accessed 13 Aug 2013).

11 Jasmer RM, Nahid P, Hopewell PC. Clinical practice. Latent tuberculosis infection. N Engl J Med 2002;347:1860-6.

12 Behar SM, Shin DS, Maier A, et al. Use of the T-SPOT.TB assay to detect latent tuberculosis infection among rheumatic disease patients on immunosuppressive therapy. J Rheumatol 2009;36:546-51.

13 Ponce de Leon D, Acevedo-Vasquez E, Alvizuri S, et al. Comparison of an interferon-gamma assay with tuberculin skin testing for detection of tuberculosis (TB) infection in patients with rheumatoid arthritis in a TB-endemic population. J Rheumatol 2008;35:776-81.

14 Martin J, Walsh C, Gibbs A, et al. Comparison of interferon \{gamma\} release assays and conventional screening tests before tumour necrosis factor \{alpha\} blockade in patients with inflammatory arthritis. Ann Rheum Dis 2010;69:181-5.

15 Bocchino $M$, Matarese A, Bellofiore B, et al. Performance of two commercial blood IFN-gamma release assays for the detection of Mycobacterium tuberculosis infection in patient candidates for anti-TNF-alpha treatment. Eur J Clin Microbiol Infect Dis 2008;27:907-13.

16 Kleinert S, Kurzai 0, Elias J, et al. Comparison of two interferon-gamma release assays and tuberculin skin test for detecting latent tuberculosis in patients with immune-mediated inflammatory diseases. Ann Rheum Dis 2010;69:782-4.
17 Matulis G, Juni P, Villiger PM, et al. Detection of latent tuberculosis in immunosuppressed patients with autoimmune diseases: performance of a Mycobacterium tuberculosis antigen-specific interferon gamma assay. Ann Rheum Dis 2008;67:84-90.

18 Martyn-Simmons CL, Mee JB, Kirkham BW, et al. Evaluating the use of the interferon-gamma response to Mycobacterium tuberculosis specific antigens in patients with psoriasis prior to anti-tumour necrosis factor alpha therapy: a prospective head-to-head cross sectional study. Br J Dermatol 2012;168:1012-8.

19 Pareek M, Bond M, Shorey J, et al. Community-based evaluation of immigrant tuberculosis screening using interferon gamma release assays and tuberculin skin testing: observational study and economic analysis. Thorax 2013;68:230-9.

20 Public Health Agency of Canada. Canadian tuberculosis standards. Ottawa 2007 [cited 2012 November]; http://www.phave-aspc.gc.ca/tbpc-lath/pubs/pdf/tbstand07_ e.pdf\%5D.

21 Pedrazzoli D, Fulton N, Anderson L, et al. Tuberculosis section, Health Protection Services, HPA. Tuberculosis in the UK: 2012 report. www.hpa.org.uk/Publications/ InfectiousDseases/Tuberculosis/1206TBintheUK2012report (accessed 13 Aug 2013).

22 Ponce de Leon D, Acevedo-Vasquez E, Sanchez-Torres A, et al. Attenuated response to purified protein derivative in patients with rheumatoid arthritis: study in a population with a high prevalence of tuberculosis. Ann Rheum Dis 2005:64:1360-1.

23 Cattamanchi A, Smith R, Steingart KR, et al. Interferon-gamma release assays for the diagnosis of latent tuberculosis infection in HIV-infected individuals: a systematic review and meta-analysis. J Acquir Immune Defic Syndr 2011;56:230-8.

24 Cobelens FG, Menzies D, Farhat M. False-positive tuberculin reactions due to non-tuberculous mycobacterial infections. Int J Tuberc Lung Dis 2007;11: 934-5.

25 Farhat M, Greenaway C, Pai M, et al. False-positive tuberculin skin tests: what is the absolute effect of BCG and non-tuberculous mycobacteria? Int J Tuberc Lung Dis 2006;10:1192-204.

26 Comstock GW, Livesay VT, Woolpert SF. Evaluation of BCG vaccination among Puerto Rican children. Am J Public Health 1974;64:283-91.

27 Diel R, Loddenkemper R, Nienhaus A. Evidence-based comparison of commercial interferon-gamma release assays for detecting active TB: a metaanalysis. Chest 2010;137:952-68.

28 Mulder C, van Deutekom H, Huisman EM, et al. Role of the QuantiFERON(R)-TB Gold In-Tube assay in screening new immigrants for tuberculosis infection. Eur Respir J 2012;40:1443-9.

29 Kim YJ, Kim SI, Kim YR, et al. Predictive value of interferon-gamma ELISPOT assay in HIV 1 -infected patients in an intermediate tuberculosis-endemic area. AIDS Res Hum Retroviruses 2012;28:1038-43.

30 Lalvani A, Millington KA. Screening for tuberculosis infection prior to initiation of anti-TNF therapy. Autoimmun Rev 2008;8:147-52.

31 Redelman-Sidi G, Sepkowitz KA. Interferon gamma release assays in the diagnosis of latent tuberculosis infection among immunocompromised adults. Am J Respir Crit Care Med Published Online First: 21 Dec 2012. PMID: 23262514.

32 Smith R, Cattamanchi A, Steingart KR, et al. Interferon-gamma release assays for diagnosis of latent tuberculosis infection: evidence in immune-mediated inflammatory disorders. Curr Opin Rheumatol 2011;23:377-84.

33 Singanayagam A, Sridhar S, Dhariwal J, et al. A comparison between two strategies for monitoring hepatic function during antituberculous therapy. Am J Respir Crit Care Med 2012;185:653-9.

34 Steele MA, Burk RF, DesPrez RM. Toxic hepatitis with isoniazid and rifampin. A meta-analysis. Chest 1991;99:465-71.

35 Dhasmana DJ, Nash J, Bradley JC, et al. TB screening and anti-TNFalpha treatment. Thorax 2006;61:641. 